\title{
Study of the PSF for Linear Motion Blurring at Arbitrary Direction
}

\author{
Gao Mei \\ The Civil Aviation University of China, Tianjin, China
}

\begin{abstract}
Motion-blurring is caused by the relative motion between the camera and the object. It is a ubiquitous matter in the fields of the photo shot at the plane or space aircraft and the photo of object moving at high speed and so on. The paper analyses the theory of images blurred by motion and sets up the model of image degradation and restoration. The paper also proves that the two-dimensional PSF (point spread function) must be directly set in the motion direction in order to restore image accurately and summarizes that setting PSF accurately is the key to the image restoration. The paper proposes an algorithm to set up PSF in the spatial domain, using the knowledge of computer graphics, digital image processing and numerical analysis. It solves the problem that it is not accurate to restore image in the frequency domain because of the particularity of the frequency spectrum.
\end{abstract}

KEYWORD: Uniform Linear Motion Blurring; Image Restoration; Bresenham Algorithm; Bilinear Interpolation; PSF

\section{GENERAL INSTRUCTIONS}

The motion blurring images may appear in the domain of monitoring of traffic video, reconnaissance of image in battlefield and so on. Motion blurring image makes the following process become very difficult, such as image target detection, 3D image reconstruction and feature extraction. So the study on the restoration of motion blurring image is of practical significance, and can be widely used in astronomy, military affairs, traffic, medical images and industrial controlling field. In the process of image restoration PSF (point spread function) is the most important factor. Its accuracy can affect the quality of the restored image.

For the physical problems, linear motion in any direction can be decomposed into two perpendicular directions $x$ axis and $y$ axis. So for any direction of motion blurring image, is it right? The answer is no. The motion blurring image is different from a physical problem. Image degradation process involves image information in the particular direction. If it is decomposed into two mutually perpendicular directions, the results can be different. So the PSF in the motion blurring image in any direction, can only be set in the particular direction of motion, can't be decomposed. If the PSF is set in frequency domain, the phenomenon of PSF has some values of zero in some points will appear.
Since the transfer function in frequency domain has zero values, it will affect the quality of image restoration.

According to the mechanism of motion blurring, the paper proposes an algorithm to set the PSF directly in the image motion direction based on the knowledge of computer graphics and bilinear interpolation algorithm. The PSF matrix can be acquired by using the algorithm. The algorithm has general applicability, combined with spatial domain recovery method, can accurately recover the image which have linear motion in any direction. It made the application range of the image restoration method become more widely.

\section{DEGRADATION MODEL OF MOTION BLURRING IMAGE}

The original image is represented by $g(x, y)$, after a degradation process represented by $H$, and then adding a noise of $z(x, y)$, a degraded image $f(x, y)$ will come into being. The degradation of the process can be described by the following formula.

$$
f(x, y)=H[g(x, y)]+n(x, y)
$$

The formula in the pattern of vectors is: 


$$
\mathbf{f}=\mathbf{H g}
$$

It is not considering of the noise.

In the formula, f,g represent the column vector, respectively constructed by the matrix of the rows of $f, g$. It can be found that the output of the system is uniquely determined by both the input and the PSF matrix. So the PSF is an important factor to describe the image characteristic of the system.

When establishing the degradation model of motion blurring image, it is considered that the degradation caused by the motion only.Assuming that the original image is $g(x, y)$, the angle between the motion direction and $x$ axis is $\theta$, the shift in the direction of $x$ is $x_{0}$, the shift in the direction of $y$ is $y_{0}$, the exposure time is $t_{1}$, the formula can be composed of:

$$
f(x, y)=\int_{0}^{t_{1}} g\left[x-x_{0}(t), y-y_{0}(t)\right] d t
$$

Fourier transform is applied on both sides of the formula, and according to the properties of Fourier transform, the formula can be obtained in other pattern:

$$
F(u, v)=G(u, v) H(u, v)
$$

In the formula, $H(u, v)$ is

$$
H(u, v)=\int_{0}^{t_{1}} \exp \left[-i 2 \pi\left(u x_{0}(t)+v y_{0}(t)\right)\right] d t
$$

$H(u, v)$ is the frequency domain representation of PSF.

Further simplification of the expression of $H(u, v)$ is:

$$
\begin{aligned}
& H(u, v)=t_{1} \sin \left(\pi \Omega V t_{1}\right) \\
& \Omega=u \cos \left(90^{\circ}+\theta\right)+v \sin \left(90^{\circ}+\theta\right)
\end{aligned}
$$

From the equation it can be found that there is a dark line in the direction of angle of $\theta+90^{\circ}$. It means that the value is zero in the points. When it is anti-transformed the PSF in spatial domain can be obtained.

Assuming that the target is of uniform motion only in $x$ axis. When the time is equal with $t_{1}$, the total distance of the image is represented by $a$. The shift in the direction of $x$ axis is:

$$
x_{0}(t)=\frac{a t}{t_{1}}, y_{0}(t)=0 \text {. }
$$

The blurred image of $f(x, y)$ is transformed into:

$$
f(x)=\int_{0}^{t_{1}} g\left[x-x_{0}(t)\right] d t=\int_{0}^{t_{1}} g\left(x-a t / t_{1}\right) d t
$$

$t^{\prime}=a t / t_{1}$, then the formula is

$$
f(x)=\int_{0}^{t_{1}} g\left(x-t^{\prime}\right) \frac{t_{1}}{a} d t^{\prime}=g(x) \otimes h(x)
$$

In the formula

$$
h(x)=\frac{t_{1}}{a}, \quad 0 \leq x \leq a
$$

The above formula is the PSF of motion blurring image in spatial domain which is of uniform motion in the direction of $x$ axis only.

\section{BRESENHAM ALGORITHM OF COMPUTER GRAPHICS}

For the uniform motion blurring in any direction, its PSF is established on the basis of the knowledge of computer graphics. In the algorithm it is also involved with bilinear interpolation algorithm. To get PSF, the first step is to find every point on the line which represents the motion direction. The algorithm of the generating line is applied to the step. Bresenham algorithm has been chosen in many generating line algorithms.

For the grating scanner, point (pixel) is the most basic element. Here the point is different from the geometric point. Geometric point has only position information and no value information, and the coordinate of the points can be arbitrary real values. The pixel has certain value and the coordinate often takes a positive integer. It cannot accurately draw a straight line between the two given points. It can be represented by the pixels near the line. In the mathematical model, a line which generated by computer is represented by the coordinates which is of integer near the line.

How to choose the best position of the grating line (pixels) is the goal of the Bresenham algorithm. The basic idea of Bresenham algorithm is: according to the slope of the line in the length direction ( $x$ axis or $y$ axis), each time the coordinate increases a unit step, in the graphics it is a pixel unit, the increment in the other direction is 0 or 1 . It changes or not is determined by the error $e$, the distance between the current point and the point on the line. Advantage of this algorithm is that the calculation is simple, involving integer only.

The symbol of the error $e$ is the factor to change the coordinate or not. Assuming that $x$ axis is the maximum shift direction, the error $e$ is the distance between the line calculated in the direction parallel to the $y$ axis and the actual point. If the step is $t$, the error $e$ is $\frac{\Delta y}{\Delta x} t$. If $e<\frac{1}{2} t$, the accurate line is closer to the point below the intersection. The original point should be displayed in the first step. It means that the direction coordinate doesn't change. If $e \geq \frac{1}{2} t$, the point above the intersection should be displayed. 
Assuming that the angle between the image motion direction and the horizontal direction is $30^{\circ}$ and the movement distance is 10 pixels. Bresenham algorithm is as shown in the following matrix. In the matrix 0 represents the point isn't involved in the line and 1 means it is involved

$$
\left[\begin{array}{llllllll}
0 & 0 & 0 & 0 & 0 & 0 & 0 & 1 \\
0 & 0 & 0 & 0 & 0 & 1 & 1 & 0 \\
0 & 0 & 0 & 0 & 1 & 0 & 0 & 0 \\
0 & 0 & 0 & 1 & 0 & 0 & 0 & 0 \\
0 & 1 & 1 & 0 & 0 & 0 & 0 & 0 \\
1 & 0 & 0 & 0 & 0 & 0 & 0 & 0
\end{array}\right]
$$

This is the first step to calculating PSF.

\section{BILINEAR INTERPOLATION ALGORITHM}

Because the distances between each pixel and the actual line are different, the impacts on the formation of the line are not identical. To determine the corresponding coefficient the bilinear interpolation of gray level interpolation is adopted. In calculating the weight is used which is calculated according to the distance between every pixel to actual line.

In the interpolation operation the first step is to find the input image point corresponding to the output pixel. Then the specified output pixel gray value is calculated through calculating the mean value of the weight of the pixels around it. The weight is determined by the distance between the pixel and every point around it.

For the bilinear interpolation, assuming that the width of the output image is $W$, the height is $H$, the width of the input image is $w$, the height is $h$. According to the method of linear interpolation, the width of the input image is divided into $W$ equal parts, the height direction is divided into $H$ parts. Then the gray value of the pixel $(x, y)$ in output image pixel is determined by the gray values of the four points $(a, b),(a+1, b),(a, b+1),(a+1, b+1)$.The values of $a$ and $b$ are

$a=\left[x \times \frac{w}{W}\right], b=\left[y \times \frac{h}{H}\right] \quad 0 \leq x \leq W, 0 \leq y \leq H$

The gray value of $(x, y)$ is represented by $f(x, y)$ is

$f(x, y)=(b+2-y) f(x, b)+(y-b) f(x, b+1)$

In above formula,

$f(x, b)=(x-a) f(a+1, b)+(a+1-x) f(a, b)$

$f(x, b+1)=(x-a) f(a+1, b+1)+(a+1-x) f(a, b+1)$
Every coefficient can be calculated through the weight of the pixels by applying the bilinear interpolation algorithm to the matrix which acquired by using Bresenham algorithm. After calculating, the matrix is

$\left[\begin{array}{cccccccc}0 & 0 & 0 & 0 & 0 & 0 & 0 & 1.0000 \\ 0 & 0 & 0 & 0 & 0 & 0.8660 & 0.6340 & 0 \\ 0 & 0 & 0 & 0 & 0.7679 & 0 & 0 & 0 \\ 0 & 0 & 0 & 0.4039 & 0 & 0 & 0 & 0 \\ 0 & 0.5359 & 0.0359 & 0 & 0 & 0 & 0 & 0 \\ 0.1679 & 0 & 0 & 0 & 0 & 0 & 0 & 0\end{array}\right]$

\section{PSF MATRIX}

At the same time, taking into account that the PSF of the image degradation has a characteristic is that light energy through a system can decrease and can't increase when the system is s passive system.

Every point in the matrix is normalized, so the PSF matrix will be:

$\left[\begin{array}{cccccccc}0 & 0 & 0 & 0 & 0 & 0 & 0 & 0.2267 \\ 0 & 0 & 0 & 0 & 0 & 0.1963 & 0.1437 & 0 \\ 0 & 0 & 0 & 0 & 0.1741 & 0 & 0 & 0 \\ 0 & 0 & 0 & 0.0911 & 0 & 0 & 0 & 0 \\ 0 & 0.1215 & 0.0081 & 0 & 0 & 0 & 0 & 0 \\ 0.0385 & 0 & 0 & 0 & 0 & 0 & 0 & 0\end{array}\right]$

According to the analysis, the steps to establish the PSF of motion blurring image can be proposed as follows:

First for any particular direction, calculating the output pixel position of actual line using Blesenham algorithm.

Secondly deciding the coefficient of each corresponding point according to the distance between the pixel and the line. The bilinear interpolation algorithm is used in the calculating in this step.

Finally taking into account of the characteristics of the degradation system, the coefficients are determined by using a normalization method. Then the PSF matrix is acquired.

Assuming that the image moves 10 pixels in the horizontal direction, the PSF matrix calculated by formula 10 is:

$$
\left[\begin{array}{llllllllll}
0.1 & 0.1 & 0.1 & 0.1 & 0.1 & 0.1 & 0.1 & 0.1 & 0.1 & 0.1
\end{array}\right]
$$

According to the calculation of the algorithm the paper proposed, the result is:

$\left[\begin{array}{llllllllll}0.100 & 0.100 & 0.100 & 0.100 & 0.100 & 0.100 & 0.100 & 0.100 & 0.100 & 0.100\end{array}\right]$

You can see the two PSF matrix is the same. It proves that the algorithm to calculate PSF the paper proposed is feasible. 


\section{CONCLUSIONS}

The paper analyses the motion blurring image model, summaries that PSF is the key in image restoration. For the linear motion at arbitrary direction its PSF should be established directly in the direction and cannot be decomposed to the two mutually perpendicular directions. Since the transfer function in frequency domain has zero values in some points, it will affect the quality of the image restoration. The paper proposes an algorithm to establish PSF in spatial domain on the base of the knowledge of computer graphics and the bilinear interpolation algorithm. The steps to get PSF are given in this paper and the matrix is simple. The paper also gives some examples to calculate their PSF. The results prove that the algorithm is effective and can be applied widely. It can be used with spatial restoration method to restore image accurately. It has broaden the scope of application of image restoration methods.

\section{ACKNOWLEDGEMENT}

The author would like to thank the support of National Natural Science Funds of China (Grant No. 61102096), the projects of CAUC BJ63028 and ZXH2012P002.

\section{REFERENCES}

[1] Zhao Rong Chun, Zhao Zhongming, Cui su. Digital image processing guide theory. Xi'an: Northwestern Polytechnical University Publishing

[2] K.R.Castleman. Digital image processing. Beijing: Publishing House of Electronics Industry, 2002:324-334.

[3] Wang Xiaohong, Zhao Rongchun. Elimination of motion blurring at arbitrary direction Chinese journal of image and graphics, 2000, 5(6):525-529.

[4] Xu Fei, Shi Xiaohong. MATLAB image processing applications. Xi'an: Xi'an Electronic and Science University Press, 2002:47-53. 